\title{
Regulation Factors of Apoptosis of Ovarian Granular Cell
}

\author{
Yang Wang ${ }^{1, a}$ and Bin Wang ${ }^{2, b^{*}}$ \\ 1,2Department of Physiology of Qiqihar Medical University, Qiqihar, Heilongjiang, 161006 \\ åRachal830813@qq.com, bijcao7835@sina.com
}

Keywords: Granular cell; Apoptosis; Regulation factors

\begin{abstract}
Follicular atresia is a common phenomenon in mammalian ovary, which is caused by the apoptosis of ovarian granular cells. The status of granular cells has become an important reference index to measure the oocyte quality and embryo development ability. Therefore, it is necessary to study the apoptosis of granular cells. There are many factors that regulate granular cell apoptosis. This paper reviews the regulation factors of ovarian granular cell apoptosis from the aspect of apoptosis factors related to the apoptosis of granular cells.
\end{abstract}

\section{Introduction}

Granular cells(GC) are a very important class of cells in the ovary. They are located on the lateral side of the zona pellucida of oocytes, and usually multilayered. They are connected with oocytes by gap junctions, and play an important role in the process of oocyte maturation in vivo and in vitro[1]. The two are closely related: Oocyte can guide the proliferation and differentiation of granular cells, and the functional state of granular cells will affect the quality of oocytes and the developmental potential of embryos[2].

There is a common follicular atresia in mammalian ovaries. As the individual age and the stage of follicular development stage vary in follicular atresia, the specific process of formation of atresia is not the same. However, in the ultrastructure study of atresia follicles, it is found that there are morphologic changes of apoptosis in follicle cells, such as karyopyknosis, the appearance of vacuoles in the cytoplasm and the formation of atresia corpuscle. These changes first occur in granular cells. Further electrophoresis experiments show that the DNA fragments isolated from granular cells of atresia follicles form an obvious ladder pattern, which is also a characteristic sign of cell apoptosis. Therefore, it is widely believed that the essence of follicular atresia is the result of ovarian granular cell apoptosis. This paper reviews the regulatory factors of ovarian granular cell apoptosis from the aspect of apoptosis factors related to the apoptosis of granular cells.

\section{Apoptosis Factors Related to the Apoptosis of Granular Cells.}

Bcl-2 and Bax Gene. The first one is the Bcl-2 Gene. Bcl-2 gene B-cell lymphoma/leukemia-2 protooncogene is a widely studied cell apoptosis signal gene at present. Human bcl-2 is a oncogene cloned by Tsujimoto and others in 1984 from the breakpoint of the $t(14 ; 18)$ chromosome translocation associated with follicular lymphocytoma. Bcl-2 has a highly conservative tendency in evolution, and its protein function is relatively conservative. From protozoa to human cells, Bcl-2 has the function of inhibiting cell apoptosis. Bcl-2 protein is a membrane related protein. The hydrophobic region of $\mathrm{Bcl}-2$ protein is inserted into the membrane structure, so that the Bcl-2 molecule's spherical structure extends into the cytoplasm. It is confirmed that this insertion plays an important role for Bcl-2 molecules to prevent cell apoptosis. Bcl-2 protein can be expressed in the activation and development of normal cells. It is also expressed in mature tissues and is low or not expressed in apoptotic cells. Bcl-2 protein can exist in normal tissues such as immune, nerve and endocrine systems, and can also exist in various types of tumor tissues of the human body[3].

The second one is the Bax gene. The Bax gene, which was first reported by Oltvai in 1993, is a related protein homologous with Bcl-2, which has an antagonistic effect on the biological activity of the latter. The bax gene is a member of the Bcl-2 gene family. It is located on the human chromosome 19 q13.3-13.4, is 4.5kb long, and contains 6 exons. The encoding products of Bax 
gene in mice can encode 3 proteins: Bax- $\alpha, \operatorname{Bax}-\beta$ and Bax- $\gamma$ though different $\alpha, \beta$ and $\gamma$ splicing modes. The most common one is the encoding of Bax- $\alpha$ protein, which is composed of 192 amino acids, and the molecular weight is $21 \mathrm{~kb}$, and this protein has $21 \%$ homology with Bcl-2 protein.

The third one is the Bcl-2/Bax relationship. The mechanism of cell apoptosis is closely regulated by the members of the Bcl-2 family. Studies show that the members of the Bcl-2 family usually play a role in the form of dimer, and whether the cells are going to survive or die depends on the ratio of Bc1-2/Bax. Immunohistochemical studies showed that the expression of Bax in tissues and organs is more extensive than Bcl-2, and exists in many tissues including the intestine, lung, stomach, pleura, lymph gland, bone marrow, liver, spleen, pancreas, heart, brain, endometrium and testis. The expression concentration in men's testicles and women's ovaries is the highest. Therefore, Bax has a certain tissue specificity for the germ cells, while Bcl-2 is seldom or not expressed in the liver, kidney, pancreas and other places. Bcl-2 and Bax are a pair of mutual antagonistic genes when the protein expression in the two is out of balance. In the body, $\mathrm{Bc} 1-2$ and $\mathrm{Bax}$ can form $\mathrm{Bc} 1-2 / \mathrm{Bc} 1-2$ or Bax/Bax homodimer, and can also form $\mathrm{Bc} 1-2 / \mathrm{Bax}$ heterodime. If $\mathrm{Bax} / \mathrm{Bax}$ homodimer increases, the activity of Bcl-2 decreases, which may cause the increase of cell mitochondrial permeability and accelerate cell apoptosis[5]. On the contrary, if the content of $\mathrm{Bcl}-2$ protein is high, the $\mathrm{Bc} 1-2 / \mathrm{Bax}$ heterodimer increases, and it is more stable than Bax/Bax homodimer, and can inhibit the cell apoptosis[6]. With the further research, many homologous genes of Bcl-2 protein family are observed. They constitute a complex interaction network, regulating the occurrence of cell apoptosis[7].

The fourth one is the correlation between Bcl-2 gene and the apoptosis of oocytes and granular cells. It is generally believed that the Bcl-2 gene plays a role in the occurrence of tumor by anti apoptosis. Bcl-2 can prevent or delay apoptosis induced by $\gamma$-radiation, glucocorticoids, heat shock and a variety of chemotherapeutic drugs. In normal cells, Bcl-2 plays an important role in inhibiting cell apoptosis and prolonging cell life. The Bcl-2 gene also plays an important role in the regulation of follicular cell apoptosis. Animal experiments show that when granular cell apoptosis leads to follicular atresia, the expression product Bcl-2 protein of the apoptosis inhibiting gene is almost not detected in the granular cells[8]. The number of ovulation and litter size of transgenic mice with specific expression of $\mathrm{Bcl}-2$ in mouse ovarian granular cells were significantly higher than that of in normal mice[9]. When the Bcl-2 gene is removed from the ovaries of the mice, the effective follicles are reduced and the follicles are found without oocyte. The exogenous bcl-2 gene transfected primary and second generation granular cells of chicken follicles can promote the division of granular cells, inhibit its apoptosis and decrease the follicular atresia rate. In the perimenopausal period, the Bcl-2 mRNA of the rat model ovarian granular cells has a weak positive expression. There is no Bcl-2 mRNA gene expression in the atresia follicle granular cells, indicating that the apoptosis of the rat follicle granular cells in perimenopausal period is closely related to the expression of Bcl-2[10]. The Bcl-2 gene is also expressed in the human ovarian tissue. Studies show that its over expression can antagonize Bax, inhibit the apoptosis of theca cells and granular cells, and promote the secretion of estrogen and progestogen, thereby controlling the apoptosis of oocytes, delaying follicular atresia and improving perimenopausal syndrome.

Caspase Family. The Caspase family plays a key role in the molecular mechanism of inducing apoptosis, which is the aggregation point of multiple apoptotic pathways, and is the ultimate pathway for the execution of apoptosis. Studies confirm that Caspase- 3 in Caspase family is the most relevant to apoptosis, because it is located in the central location of receptor mediated apoptosis pathway and mitochondria mediated apoptosis pathway. The exact molecular mechanism of inducing apoptosis of granular cells is still unclear, which may be the result of the interaction between follicle stimulating factor Bcl-2 and inducible atresia factor Caspase-3. Studies have shown that the inhibitory effect of Bcl-2 and Caspase-3 is mutual, Bcl-2 can inhibit the activation of Caspase-3, and meanwhile, Bcl-2 is also one of the substrates of activated Caspase-3. Activated Caspase- 3 degradates Bcl-2 and Bcl-2 protein decreases. The expression level of Bcl-2 protein in tissue can indirectly reflect the strength and weakness of the activity of Caspase-3, that is, it reflects the strength and weakness of cell apoptosis[11]. 
Fas/Fas-L Signal Transduction Pathway. The Fas system belongs to the tumor necrosis factor receptor family, and FasL is the only natural ligand in its body. It has been believed that the apoptosis signaling pathway mediated by Fas/FasL system is one of the important pathways of cell apoptosis. The activation of Caspase family protease is one of the key links in the mechanism of apoptosis, and plays a key role in the process of apoptosis. Caspase- 3 is one of the most important proteins in Caspase family members, and is closely related to apoptosis. Caspase-3 protease is involved in multiple pathways induced apoptosis. Caspase- 8 is the promoter of the Caspase, and Caspase- 3 is divided into the executor of the Caspase. Studies have confirmed that Caspase- 3 is a core protease in Fas mediated apoptosis signal transduction pathway. Inhibiting Caspase-3 enzyme activity can inhibit the apoptotic signal pathway, which proves that Caspase- 3 is an essential enzyme for cell apoptosis. Fas mediated signal transduction pathway of apoptosis is the most critical part of TNF receptor family transduction pathway, and plays a decisive role in it. Fas exists on the target cells, and FasL is combined with it in the form of a trimer. Fas trimer is induced in order to activate the Fas-Associated Death Domain(FADD). FADD can accomplish two aspects. Firstly, it can combine Fas through DD at the $\mathrm{C}$ end. Secondly, it can recruit procaspase- 8 to Fas area through death effector domain(DED) that can cause apoptosis at its $\mathrm{N}$ end. It cuts itself and activates, thus initiating the enzyme chain reaction of the caspase family. Caspase- 3 is the ultimate performer of the enzyme chain reaction of the Caspase family, which ultimately affects the structural proteins of the cells, leading to the occurrence of cell apoptosis[12].

p53 Gene. p53 plays an important regulatory role in the normal growth of cells, which can obviously inhibit the transformation and growth of cells. Its product p53 protein can be combined with the specific DNA sequence and activates the transcription. p53 is a typical gene that promotes apoptosis, and many apoptotic factors depend on the activation of p53 to regulate apoptosis. Immunohistochemical staining shows that p53 protein is only located in the nucleus of granulosa cells with apoptosis of atresia follicles. After eCG treatment, p53 protein immunostaining decreases to an indetectable level. When there is the spontaneous apoptosis of the healthy cavity follicle cultivated in vitro, the mRNA level of p53 is significantly higher than that of the control group, which promotes the apoptosis of granular cells [13].

Myc Gene. Myc is a nuclear protein gene, which is related to cell proliferation, differentiation, regulation of cell cycle and apoptosis. The expression of Myc protein in follicles changes with the improvement of follicle development and function. In the oocyte of the primary follicle stage, the expression of Myc protein was strongly positive, and gradually decreased with the expression of follicular development, and was positive in granular cells and theca cells. In atresia follicles, the expression of Myc protein only exists in theca cells. It is concluded that Myc protein may be a factor that promotes apoptosis to induce the apoptosis of granular cells and theca cells[14].

\section{Conclusion}

The regulatory genes of apoptosis control the apoptosis of granular cells through mitochondrial pathway and death receptor pathway. They play an important role in different links of the apoptotic pathway, and there is a certain synergistic or antagonistic effect between them. The specific mechanisms need to be further studied.

The apoptosis of granular cells is not only the independent process of the regulation of the cell's own genes, but also the result of the interaction of many extracellular environmental factors. The further studies on apoptosis of follicle granular cells and its influencing factors are of great significance for revealing the essence of follicular atresia, the human control of the development of the follicle and the model of cell apoptosis study.

\section{Acknowledgements}

Science and technology planning project in Qiqihar city, Heilongjiang Province. (SFGG-201565): Influence of Yanling dan Prescription on the Apoptosis of Ovarian Granular Cells in Mice with Premature Ovarian Failure 


\section{References}

[1] Y.P.Wu, H.H.Zhao, Y.X.Zhou, etc: The Role of Granulosa Cells in the Development of Oocyte Maturation[J], Journal of International Reproductive Health/Family Planning, 2017, 36(6): 503-506.(In Chinese)

[2] B.Cai, Y.Tuo, Y.B.Li, etc: Derivation of Integration-free iPSCs from Human Mural Granulosa Cells[J], Journal of Reproductive Medicine, 2017, 26(4):313-319.(In Chinese)

[3] Wang, X, Wang Y, Kim HP, et al.Carbon monoxide protects against hyperoxia-induced endothelial cell apoptosis by inhibiting reactive oxygen species formation. J Biol Chem,2007, 282(3):1 718-26.

[4] H.Li, W.S.Zeng, S.Luo, etc: Inhibitory Effect of RNA Interference Targeting BaxBak on Apoptosis of Human Granulosa Cells[J], Journal of Southern Medical University, 2009, 29(12):2367-2370.(In Chinese)

[5] Edassery SL, Shatavi SV, Kunkel JP, et al. Autoanti-gens in ovarian autoimmunity associated with unexplained infertility and premature ovarian failure[J].Fertil Steril , 2010 , 94(7):2636-2641.

[6] Z.Liu and J.Zheng: Bcl-2 Family Protein and Its Role in Cell Apoptosis[J], Chemistry of Life, 2007, 27(1):22-25.(In Chinese)

[7] S.Z.Qin: The Mechanism and Application of the Interaction of Bcl-2 Family Protein[J], Chinese Medicinal Biotechnology, 2007, 2(3):218-221.(In Chinese)

[8] Y.Li, M.Y.Wang and H.Ma: Regulation Mechanism of Ovarian Granulosa Cell Apoptosis[J], Journal of Clinical and Experimental Medicine, 2007, 6(4):160-161.(In Chinese)

[9] H.P.Liu, Z.L.You, L.Huang, etc: Effect of Bushenhuoxue Prescription on Bcl-2/Bax Protein of Apoptosis Contress Genes in Follicle of Immune Prenature Ovarian Failure Mice[J], Chineses Archives of Traditonal Chinese Medicine, 2010, 28(5):1036-1038.(In Chinese)

[10] K.K.Zhao, L.Gao, K.Xue, etc: Effect and Mechanism of Androgen on Cultured Mouse Ovarian Granulosa Cell Apoptosis[J], Jinagsu Medical Journal, 2011, 37(16):1861-1865.(In Chinese)

[11] M.Zhang, X.X.Qiu, C.L.Zhu, etc: The Effect of Bushenyichongkangshuai Decoction on Ovarian Granule Cell Apoptosis Factors Bcl-2,Bax,Caspase-3 of Dor Rat Model[J], Journal o fZhejiang University of Traditional Chinese Medicine, 2014, 38(1): 68-72.(In Chinese)

[12] J.Mao, L.L.Wang, Y.Li, etc: Effects of Bufei Jianpi Formula on the Expressions of Fas, FasL, Bcl-2 and Bax in Skeletal Muscle of Rats with COPD[J], China Journal of Traditional Chinese Medicine and Pharmacy, 2017, 32(2): 746-749.(In Chinese)

[13] H.Z.Cui, K.M.Zhang and L.Wang: Research Trends of Epigenetic Mechanism of p53 Pathway in Psoriasis[J], Chinese Journal of Dermatovenereology of Integrated Traditional and Western Medicine, 2010, 9(6): 395-397.(In Chinese)

[14] Y.L.Zhou, H.Y.Zheng, Y.Liu: Research Progress of MYC[J], Journal of Gannan Medical University, 2017, 37(1): 140-145.(In Chinese)

\section{Corresponding author}

Bin Wang, Doctor degree candidate. Work in Department of Physiology of Qiqihar Medical University, Qiqihar, Heilongjiang, 161006 